\title{
Pricing Parisian Option under a Stochastic Volatility Model
}

\author{
Min-Ku Lee ${ }^{1}$ and Kyu-Hwan Jang ${ }^{2}$ \\ ${ }^{1}$ Department of Mathematics, Sungkyunkwan University, Suwon, Gyeonggi-do 440-746, Republic of Korea \\ ${ }^{2}$ Department of Mathematics, Yonsei University, Seoul 120-749, Republic of Korea \\ Correspondence should be addressed to Kyu-Hwan Jang; v5jang@hanmail.net
}

Received 5 June 2014; Revised 29 September 2014; Accepted 29 September 2014; Published 19 November 2014

Academic Editor: Roberto A. Kraenkel

Copyright (C) 2014 M.-K. Lee and K.-H. Jang. This is an open access article distributed under the Creative Commons Attribution License, which permits unrestricted use, distribution, and reproduction in any medium, provided the original work is properly cited.

\begin{abstract}
We study the pricing of a Parisian option under a stochastic volatility model. Based on the manipulation problem that barrier options might create near barriers, the Parisian option has been designed as an extended barrier option. A stochastic volatility correction to the Black-Scholes price of the Parisian option is obtained in a partial differential equation form and the solution is characterized numerically.
\end{abstract}

\section{Introduction}

The Black-Scholes model is a well-known model in derivatives pricing under which volatility is assumed to be a constant [1]. From empirical studies based on real market data, one can observe skew or smile effect of implied volatilities, fat-tailed and asymmetric return distributions, and meanreversion of volatility. Thus to overcome these unrealistic phenomena many alternative underlying models have been proposed. The constant elasticity of variance model by Cox [2], a stochastic volatility model by Heston [3], a multiscale stochastic volatility model by Fouque et al. [4], and a Levy model by Carr et al. [5] are popular ones among many others.

A basic standard option gives its holders the right to sell or buy a stock for a designated amount of money before or at a maturity time. However, there are many kinds of options that have been developed recently in such way that the payoff of options becomes more complicated. The barrier option is one of those exotic options where the payoff of the option depends on the path of its underlying asset. The barrier option can be exercised if the value of underlying asset hits the barrier during a time period before the maturity. For instance, the barrier option of knockout type corresponds to a vanilla European option if the value of an underlying asset does not hit the barrier $B$ before the maturity $T$. However, the price of option immediately becomes worthless if the value of the underlying asset hits the barrier $B$. While this barrier option has some advantage in that it can lower the price than the vanilla option and it can be hedged cheaply for the risk of underlying assets, it creates some problems. First, there is a manipulation problem. Some hedge funds or investors can make an intentional manipulation of the barrier option for their profit. Since the payoff of the barrier option is decided only by whether the value of an underlying asset touches the barrier or not, if some hedge funds intentionally buy and sell a great quantity of the underlying asset, it is possible for the underlying asset artificially to touch the barrier. Second, there is a hedging problem. If an underlying asset approaches the barrier $B$, then Gamma in Greeks becomes very large and then the price of the barrier option may become distorted by the high Gamma value.

In order to solve these problems, the Parisian option has been suggested. The payoff of the Parisian option depends on how much time the value of an underlying asset keeps over/below the barrier. The value of the underlying asset hits the barrier $B$ and it must keep over the barrier for the predecided time $D$ in order to give rise to a positive payoff. This paper is interested in the pricing of this Parisian option. There are previous studies on the Parisian options. Haber et al. [6] gained the price in the form of a partial differential equation. Marco and Lixin [7] used the binomial tree method to price the option. Labart and Lelong in [8] used Laplace transformation approach for the pricing of the option. However, these works have not involved the pricing of 
Parisian option under stochastic volatility models directly. So, this paper extends the study of [6] using a stochastic volatility model, that is, the multiscale model of [4] for the pricing of a Parisian option.

In Section 2, we recall a study for the price of a Parisian option under the Black-Scholes model. In Section 3, we formulate a stochastic volatility model and obtain an approximate price of the Parisian option in the form of a partial differential equation. In Section 4, we compute the leading order price and a correction term by the finite difference method and find a stochastic volatility effect on the BlackScholes price.

\section{The Black-Scholes Model}

In this section, we review the Parisian option price under the Black-Scholes model. Refer to Haber et al. [6] and Zhu and Chen [9]. As Parisian option is a path-dependent option like barrier option, the price depends on the path of an underlying asset as well as payoff at the maturity.

To measure how much time the underlying asset price keeps over or under the barrier, a new state variable $\tau_{t}$ (a barrier time) has to be introduced. With the state variable $\tau_{t}$ we do not need to know all the specific contents of the path taken. In the case of Up and In, the state variable $\tau_{t}$ is counted when the underlying asset price has been over the barrier in the current excursion. Given the value $X_{t}$ of an underlying asset, $\tau_{t}$ is defined by

$$
\tau_{t}=t-\sup \left\{t^{\prime} \leq t \mid X\left(t^{\prime}\right) \leq B\right\} .
$$

So, in the Up and In option case, the barrier time $\tau_{t}$ gives the difference between the current time $t$ and the last time when the underlying asset price is less than the barrier $B$. If the underlying asset price is presently below the barrier, then $\tau_{t}$ is zero.

In the Down and In option case, the variable $\tau_{t}$ is opposite to the case in the Up and In option. The state variable $\tau_{t}$ is defined by

$$
\tau_{t}=t-\sup \left\{t^{\prime} \leq t \mid X\left(t^{\prime}\right) \geq B\right\} .
$$

In the Down and In option case, the barrier time $\tau_{t}$ gives the difference between the current time $t$ and the last time when the underlying asset price is bigger than the barrier $B$. If the underlying asset is presently over the barrier, then $\tau_{t}$ is zero. by

In the Down and In option case, dynamics of $\tau_{t}$ are given

$$
d \tau_{t}= \begin{cases}d t, & \text { if } X_{t}>B \\ -\tau(t-), & \text { if } X_{t}=B \\ 0, & \text { if } X_{t}<B\end{cases}
$$

where $\tau(t-)$ denotes the left limit of $\tau_{t}$. The barrier time rises at the same rate with $t$ if the share price $X_{t}$ is over the barrier $B$ (i.e., $X_{t}>B$ ). As soon as the underlying asset price hits the barrier $B$ (i.e., $X_{t}=B$ ), it is reset to zero. Also, if the underlying asset price is below the barrier $B$ (i.e., $X_{t}<B$ ), then it does not change. The state variable $\tau_{t}$ can be viewed as a time that starts ticking as soon as the underlying asset crosses the barrier level $B$.

Additionally, we need a new variable $D$ which is called a threshold time. If the barrier time $\tau_{t}$ is more than the threshold time $D$, the Parisian option becomes an effective option.

Under the Black-Scholes model

$$
d X_{t}=r X_{t} d t+\sigma X_{t} d W_{t}^{*}
$$

where $r$ is an riskless interest rate and $W_{t}^{*}$ is a Brownian motion under a risk-neutral measure $Q$, the price $P$ of a Parisian option is a function depending on the state variables $X_{t}, t$, and $\tau_{t}$ (i.e., $P=P\left(t, X_{t}, \tau_{t}\right)$ ). The price $P$ of the Parisian option satisfies two different partial differential equations depending on whether the current underlying asset is over or under the barrier $B$. For example, let us consider the Up and Out Parisian option. If the underlying asset is below the barrier (i.e., $X_{t}<B$ ), the barrier time variable $\tau_{t}$ is not ticking and remains unchanged. Then given $X_{t}=x$ and $\tau_{t}=\tau$ the price $P$ satisfies the Black-Scholes equation given by

$$
\frac{\partial P}{\partial t}+\frac{1}{2} \sigma^{2} x^{2} \frac{\partial^{2} P}{\partial x^{2}}+r\left(x \frac{\partial P}{\partial x}-P\right)=0 .
$$

On the other hand, if the underlying asset rises over the barrier (i.e., $X_{t}>B$ ) and so the barrier time $\tau_{t}$ is ticking, the barrier time $\tau_{t}$ is valid. Then the price $P$ must have the derivative with respect to the variable $\tau_{t}$ so that it satisfies a new partial differential equation given by

$$
\frac{\partial P}{\partial t}+\frac{1}{2} \sigma^{2} x^{2} \frac{\partial^{2} P}{\partial x^{2}}+r\left(x \frac{\partial P}{\partial x}-P\right)+\frac{\partial P}{\partial \tau}=0 .
$$

Here, the price of the Parisian option is defined differently in each domain of $X_{t}>B$ and $X_{t}<B$. So, to give the continuity of $P$ at $x=B$, we define

$$
P(t, B, \tau)=P(t, B, 0) .
$$

Now, we give a boundary condition for each case (In or Out). First, we use functions $F(x, \tau)$ and $G(x)$ to define

$$
\begin{aligned}
& P(T, x, \tau)=: F(x, \tau), \quad 0 \leq \tau<D, \\
& P(t, x, D)=: G(x), \quad 0 \leq t \leq T .
\end{aligned}
$$

If at the maturity $T$ the barrier time $\tau_{t}$ is less than $D$, then the Out option has the same payoff $(x-K)^{+}$as the European option payoff, while the payoff of the In option is zero. When the underlying asset touches the barrier $B$ and the barrier time $\tau$ also keeps on the situation $\tau \geq D$, the payoff of the Out option is zero while the In option has the payoff $(x-K)^{+}$. So, the boundary conditions are given by

$$
\begin{aligned}
& F(x, \tau)= \begin{cases}(x-K)^{+}, & \text {Out, } \\
0, & \text { In, }\end{cases} \\
& G(x)= \begin{cases}0, & \text { Out } \\
(x-K)^{+}, & \text {In } .\end{cases}
\end{aligned}
$$




\section{Stochastic Volatility}

3.1. A Model Formulation. In this section, we use a stochastic volatility model of Fouque et al. of [4] for an underlying asset of the Up and Out Parisian call option. In this stochastic volatility model, the volatility is assumed to be given by an Ornstein-Uhlenbeck process which is a kind of ergodic process. The stochastic volatility model is given by the stochastic differential equations (SDEs)

$$
\begin{aligned}
& d X_{t}=r X_{t} d t+f\left(Y_{t}\right) X_{t} d W_{t}^{x}, \\
& d Y_{t}=\left(\frac{1}{\epsilon}\left(m-Y_{t}\right)-\frac{\nu \sqrt{2}}{\sqrt{\epsilon}} \Lambda\right) d t+\frac{\nu \sqrt{2}}{\sqrt{\epsilon}} d W_{t}^{y}
\end{aligned}
$$

under a risk-neutral measure $Q$, where $r$ is a constant and $f$ is a function depending on the variable $y$. Here, the market price of volatility $\Lambda$ is assumed to be independent of $x$ and $y$. $W_{t}^{x}$ and $W_{t}^{y}$ are correlated Brownian motions such that $d\left\langle W^{x}, W^{y}\right\rangle_{t}=\rho(t) d t$ and $\rho$ is a function depending on time $t$. If $\rho(t)$ is positive, $X_{t}$ may fail to be a true martingale since $Y_{t}$ may explode to infinite. Analyzing the most financial data, except in some commodity markets, the negative correlation or leverage effect between stock price and volatility of stocks is observed. So, in this paper, $\rho(t)$ is assumed to be negative. Also, for simplicity and from empirical data observed in practical situation, we assume that $\rho$ is a constant. Refer to [10]. The function $f$ is not limited to specific functions. But, to avoid the nonexistence of moments of $X_{t}$, we assume $f$ to be a function satisfying the condition $0<c_{1} \leq f \leq c_{2}<\infty$ for some positive constants $c_{1}$ and $c_{2}$.

From now on, we assume that the parameter $\epsilon$ is positive and small such that $0<\epsilon \ll 1$. Then the Ornstein-Ulhenbeck process $Y_{t}$ becomes a fast mean-reverting process. From the empirical analysis for financial data of high-frequency S\&P 500 index, one can observe that volatility is fast meanreverting when looked at over the time scale of contingent claims. Refer to [11]. The S\&P 500 index data yield that $\epsilon^{-1}$ is large and $v^{2}$ is a stable such that it is an $\mathcal{O}(1)$ constant which means that the long run magnitude of volatility fluctuations remains fixed as a constant. The parameters $m$ and $v$ stand for the mean level and the standard deviation of the invariant distribution of $Y_{t}$, respectively. The probability density function of $Y_{t}$ is given by

$$
\Phi(y)=\frac{1}{\sqrt{2 \pi v^{2}}} e^{-(y-m)^{2} / 2 v^{2}}
$$

so that the two parameters control the long-run size of the volatility fluctuation.

3.2. A Partial Differential Equation. In this paper, we derive a partial differential equation for the Parisian call option (especially, Up and Out type) based on the stochastic volatility model discussed in Section 3.1.

The price of the Parisian call option is given by

$$
P^{\epsilon}(t, x, y, \tau)=\mathbb{E}^{*}\left\{e^{-r(T-t)} h\left(X_{T}\right) \mid X_{t}=x, Y_{t}=y, \tau_{t}=\tau\right\},
$$

where

$$
h\left(X_{T}\right)= \begin{cases}\left(X_{T}-K\right)^{+} \mathbb{1}_{\left\{\exists t \leq 0 \text { s.t. } t \leq T, \tau_{t} \geq D\right\}}, & \text { if In, } \\ \left(X_{T}-K\right)^{+} \mathbb{1}_{\left\{0 \leq \forall t \leq T, \tau_{t}<D\right\}}, & \text { if Out. }\end{cases}
$$

Here, the price of the Parisian call option (12) has the domain given by

$$
\begin{gathered}
\mathscr{H}_{1}:\{(t, x, y, \tau): B \leq x<\infty,-\infty<y<\infty, \\
0 \leq t \leq T, 0 \leq \tau \leq D\}, \\
\mathscr{H}_{2}:\{(t, x, y, \tau): 0 \leq x \leq B,-\infty<y<\infty, \\
0 \leq t \leq T, \tau=0\} .
\end{gathered}
$$

Given the barrier time $\tau$, it is not possible that the elapsed time $t$ is less than barrier time $\tau$ in both domains. Also, the Parisian option cannot be exercised if the time to maturity $T-t$ is less than the remaining time to barrier $D-\tau$. So, we do not care about the regions $t<\tau$ and $T-D+\tau<t$ for a given $\tau$. Thus we have the reduced regions $\mathscr{A}_{1}$ and $\mathscr{A}_{2}$ given by

$$
\begin{gathered}
\mathscr{A}_{1}:\{(t, x, y, \tau): B \leq x<\infty,-\infty<y<\infty, \\
\tau \leq t \leq \tau+T-D, 0 \leq \tau \leq D\}, \\
\mathscr{A}_{2}:\{(t, x, y, \tau): 0 \leq x \leq B,-\infty<y<\infty, \\
0 \leq t \leq T-D, \tau=0\} .
\end{gathered}
$$

See [9] for details. For convenience, we denote $P^{\epsilon}(t, x, y, \tau)$ separately by $U^{\epsilon}(t, x, y, \tau)$ and $V^{\epsilon}(t, x, y)$ in the regions $\mathscr{A}_{1}$ and $\mathscr{A}_{2}$, respectively. That is,

$$
P^{\epsilon}(t, x, y, \tau)= \begin{cases}U^{\epsilon}(t, x, y, \tau), & \text { on } \mathscr{A}_{1}, \\ V^{\epsilon}(t, x, y), & \text { on } \mathscr{A}_{2} .\end{cases}
$$

Now, we construct the boundary condition on the domains $\mathscr{A}_{1}$ and $\mathscr{A}_{2}$. First of all, whenever the value of an underlying asset goes to zero, the worth of call option disappears. So, when the underlying price goes to zero, we can set the option price as follows:

$$
\lim _{x \rightarrow 0} V^{\epsilon}(t, x, y)=0 .
$$

Also, for the continuity of the option prices $U^{\epsilon}$ and $V^{\epsilon}$ defined on the different domains $\mathscr{A}_{1}$ and $\mathscr{A}_{2}$, respectively, we set the following condition at the barrier $B$ :

$$
\lim _{x \rightarrow B} V^{\epsilon}(t, x, y)=\lim _{x \rightarrow B} U^{\epsilon}(t, x, y, 0) .
$$

Moreover, if the underlying asset is too large to fall back to the barrier $B$ before the maturity $T$, the option can be exercised. So, in the case that the underlying asset price is infinitely large, we have

$$
\lim _{x \rightarrow \infty} U^{\epsilon}(t, x, y, \tau)=0 .
$$

Furthermore, since we cover the case of the Out option, the option value disappears once the accumulated time $\tau$ over the 
barrier $B$ becomes $D$. So, the option value near the $\tau=D$ vanishes such that

$$
\lim _{\tau \rightarrow D} U^{\epsilon}(t, s, y, \tau)=0 .
$$

Now, applying the Feynman-Kac formula to the Parisian option price (12) with the boundary condition (8), we obtain a partial differential equation in each of the regions $\mathscr{A}_{1}$ and $\mathscr{A}_{2}$, respectively, as follows:

$$
\begin{gathered}
\left(\frac{1}{\epsilon} \mathscr{L}_{0}+\frac{1}{\sqrt{\epsilon}} \mathscr{L}_{1}+\mathscr{L}_{\operatorname{Par}(f(y))}\right) U^{\epsilon}(t, x, y, \tau)=0, \\
U^{\epsilon}(T, x, y, \tau)=(x-K)^{+}, \quad 0 \leq \tau<D \\
U^{\epsilon}(t, x, y, D)=0, \quad 0 \leq t \leq T, \\
\left(\frac{1}{\epsilon} \mathscr{L}_{0}+\frac{1}{\sqrt{\epsilon}} \mathscr{L}_{1}+\mathscr{L}_{\mathrm{BS}(f(y))}\right) V^{\epsilon}(t, x, y)=0, \\
V^{\epsilon}(T, x, y)=(x-K)^{+},
\end{gathered}
$$

where

$$
\begin{gathered}
\mathscr{L}_{0}=v^{2} \frac{\partial^{2}}{\partial y^{2}}+(m-y) \frac{\partial}{\partial y} \\
\mathscr{L}_{1}=\sqrt{2} \rho \nu x f(y) \frac{\partial^{2}}{\partial x \partial y}-\sqrt{2} \nu \Lambda \frac{\partial}{\partial y}, \\
\mathscr{L}_{\mathrm{BS}(f(y))}=\frac{\partial}{\partial t}+\frac{1}{2} f(y)^{2} x^{2} \frac{x^{2}}{\partial x^{2}}+r\left(x \frac{\partial}{\partial x}-\cdot\right), \\
\mathscr{L}_{\operatorname{Par}(f(y))}=\frac{\partial}{\partial \tau}+\mathscr{L}_{2(f(y))} .
\end{gathered}
$$

3.3. Asymptotic Analysis. In this section, we approximate the price of the Parisian option satisfying the partial differential equations (21) by using the expansions

$$
\begin{aligned}
U^{\epsilon}(t, x, y, \tau) & =\sum_{i=0}^{\infty} \epsilon^{i / 2} U_{i}(t, x, y, \tau), \\
V^{\epsilon}(t, x, y) & =\sum_{i=0}^{\infty} \epsilon^{i / 2} V_{i}(t, x, y) .
\end{aligned}
$$

Putting the asymptotic expansions (23) into (21), we have the following asymptotic partial differential equations given by

$$
\begin{aligned}
& \frac{1}{\epsilon} \mathscr{L}_{0} U_{0}+\frac{1}{\sqrt{\epsilon}}\left(\mathscr{L}_{0} U_{1}+\mathscr{L}_{1} U_{0}\right) \\
& \quad+\mathscr{L}_{0} U_{2}+\mathscr{L}_{1} U_{1}+\frac{\partial U_{0}}{\partial l}+\mathscr{L}_{\operatorname{Par}(f(y))} U_{0} \\
& \quad+\sqrt{\epsilon}\left(\mathscr{L}_{0} U_{3}+\mathscr{L}_{1} U_{2}+\frac{\partial U_{1}}{\partial l}+\mathscr{L}_{\operatorname{Par}(f(y))} U_{1}\right) \\
& \quad+\cdots \\
& =0
\end{aligned}
$$

$$
\begin{aligned}
\frac{1}{\epsilon} \mathscr{L}_{0} V_{0} & +\frac{1}{\sqrt{\epsilon}}\left(\mathscr{L}_{0} V_{1}+\mathscr{L}_{1} V_{0}\right) \\
& +\mathscr{L}_{0} V_{2}+\mathscr{L}_{1} V_{1}+\frac{\partial V_{0}}{\partial l}+\mathscr{L}_{\mathrm{BS}(f(y))} V_{0} \\
& +\sqrt{\epsilon}\left(\mathscr{L}_{0} V_{3}+\mathscr{L}_{1} V_{2}+\frac{\partial V_{1}}{\partial l}+\mathscr{L}_{\mathrm{BS}(f(y))} V_{1}\right) \\
& +\cdots \\
= & 0 .
\end{aligned}
$$

Additionally, the boundary conditions for each function of $U_{i}$ and $V_{i}(i=0,1,2, \ldots)$ become

$$
\begin{gathered}
U_{0}(T, x, y, \tau)=(x-K)^{+}, \quad 0 \leq \tau<D, \\
U_{0}(t, x, y, D)=0, \quad 0 \leq t \leq T, \\
U_{i}(T, x, y, \tau)=0, \quad 0 \leq \tau<D(i=1,2, \ldots), \\
U_{i}(t, x, y, D)=0, \quad 0 \leq t \leq T(i=1,2, \ldots), \\
V_{0}(T, x, y)=(x-K)^{+}, \\
U_{i}(T, x, y)=0 . \quad(i=1,2, \ldots) .
\end{gathered}
$$

Now, we derive the leading order term $U_{0}$ and the correction term $U_{1}$ in a partial differential equation form. From (24), we have

$$
\begin{aligned}
& \frac{1}{\epsilon}: \mathscr{L}_{0} U_{0}=0, \\
& \frac{1}{\sqrt{\epsilon}}: \mathscr{L}_{0} U_{1}+\mathscr{L}_{1} U_{0}=0, \\
& 1: \mathscr{L}_{0} U_{2}+\mathscr{L}_{1} U_{1}+\mathscr{L}_{\operatorname{Par}(f(y))} U_{0}=0, \\
& \sqrt{\epsilon}: \mathscr{L}_{0} U_{3}+\mathscr{L}_{1} U_{2}+\mathscr{L}_{\operatorname{Par}(f(y))} U_{1}=0 .
\end{aligned}
$$

In the following theorems, we derive partial differential equations for $U_{0}$ and $U_{1}$ on the region $\mathscr{A}_{1}$ and $V_{0}$ and $V_{1}$ on the region $\mathscr{A}_{2}$, respectively.

Theorem 1. Suppose that $U_{i},(i=0,1,2, \ldots)$ does not grow as much as $U_{i} \sim e^{y^{2} / 2}$ as $y$ goes to $\infty$. Then, the leading order term $U_{0}$ and the correction term $U_{1}$ do not depend on the variable $y$; that is,

$$
U_{i}(t, x, y, \tau)=U_{i}(t, x, \tau), \quad i=0,1 .
$$

Suppose that $V_{i}(i=0,1,2, \ldots)$ does not grow as much as $V_{i} \sim$ $e^{y^{2} / 2}$ as $y$ goes to $\infty$. Then, the leading order term $V_{0}$ and the correction term $V_{1}$ do not depend on the variable $y$; that is,

$$
V_{i}(t, x, y)=V_{i}(t, x), \quad i=0,1 .
$$

Proof. As (28) is an ordinary differential equation with respect to $y$, one can obtain the solution easily given by

$$
P_{0}(t, x, y, \tau)=c_{1}(t, x, \tau) \int_{0}^{y} e^{\left((z-m)^{2} / 2 v^{2}\right) d z}+c_{2}(t, x, \tau)
$$


for some functions $c_{1}$ and $c_{2}$ independent of $y$. Then, from the assumed growth condition, $U_{0}$ is independent of the variable $y$. Since $U_{0}$ is independent of $y$ and each term of $\mathscr{L}_{1}$ contains derivative with respect to $y$, we have $\mathscr{L}_{1} U_{0}=0$. So, $U_{1}$ is independent of $y$ from the order $1 / \sqrt{\epsilon}$ term (29).

Similarly, we have the results for $V_{0}$ and $V_{1}$. for $U_{0}$

The following theorem gives a partial differential equation

Theorem 2. The leading order term $U_{0}$ is the solution of a partial differential equation problem given by

$$
\begin{gathered}
\mathscr{L}_{\operatorname{Par}(\bar{\sigma})} U_{0}(t, x, \tau)=0, \\
U_{0}(T, x, \tau)=(x-K)^{+}, \quad 0 \leq \tau<D, \\
U_{0}(t, x, D)=0, \quad 0 \leq t \leq T,
\end{gathered}
$$

where

$$
\bar{\sigma}=\sqrt{\left\langle f^{2}\right\rangle}=\sqrt{\int f^{2}(y) \frac{1}{\sqrt{2 \pi \nu}} e^{-(y-m)^{2} / 2 v^{2}} d y .}
$$

The leading order term $V_{0}$ is the solution of a partial differential equation problem given by

$$
\begin{gathered}
\mathscr{L}_{B S(\bar{\sigma})} V_{0}(t, x)=0, \\
V_{0}(T, x)=(x-K)^{+} .
\end{gathered}
$$

Proof. Since $U_{1}$ is independent of $y$ and every term of $\mathscr{L}_{1}$ contains derivative with respect to the variable $y, \mathscr{L}_{1} U_{1}=0$ holds and so the $\mathcal{O}(1)$ term (30) becomes

$$
\mathscr{L}_{0} U_{2}+\mathscr{L}_{\operatorname{Par}(f(y))} U_{0}=0
$$

Thus we obtain a partial differential equation for $U_{2}$ which is the Poisson equation with respect to the operator $\mathscr{L}_{0}$. Applying the centering condition, we obtain

$$
\begin{aligned}
\left\langle\mathscr{L}_{\operatorname{Par}(f(y))} U_{0}\right\rangle & =\left(\frac{\partial}{\partial \tau}+\left\langle\mathscr{L}_{\mathrm{BS}(f(y))}\right\rangle\right) U_{0} \\
& =\left(\frac{\partial}{\partial \tau}+\mathscr{L}_{\mathrm{BS}(\bar{\sigma})}\right) U_{0}=\mathscr{L}_{\operatorname{Par}(\bar{\sigma})} U_{0}=0 .
\end{aligned}
$$

By adding the boundary condition (26) to this equation, the result for $U_{0}$ is derived.

Similarly, $V_{0}$ satisfies the problem (37).

Now, we derive the correction term $V_{1}$ in the form of a partial differential equation satisfied by the term.

Theorem 3. The correction term $U_{1}$ is the solution of a partial differential equation problem given by

$$
\begin{gathered}
\mathscr{L}_{\operatorname{Par}(\bar{\sigma})} U_{1}(t, x, \tau)=W_{2} x^{2} \frac{\partial^{2} U_{0}}{\partial x^{2}}+W_{3} x^{3} \frac{\partial^{3} U_{0}}{\partial x^{3}} \\
U_{1}(T, x, \tau)=0, \quad 0 \leq \tau<D, \\
U_{1}(t, x, D)=0, \quad 0 \leq t \leq T,
\end{gathered}
$$

where

$$
\begin{gathered}
W_{2}=\sqrt{2} \rho \nu\left\langle f \phi^{\prime}\right\rangle-\frac{\sqrt{2}}{2} \nu\left\langle\Lambda \phi^{\prime}\right\rangle, \\
W_{3}=\frac{\sqrt{2}}{2} \rho \nu\langle f \phi\rangle .
\end{gathered}
$$

The correction term $V_{1}$ satisfies a partial differential equation problem given by

$$
\begin{gathered}
\mathscr{L}_{B S(\bar{\sigma})} V_{1}(t, x)=W_{2} x^{2} \frac{\partial^{2} V_{0}}{\partial x^{2}}+W_{3} x^{3} \frac{\partial^{3} V_{0}}{\partial x^{3}}, \\
V_{1}(T, x)=0 .
\end{gathered}
$$

Proof. From (38) and (39), we have a Poisson equation for $U_{2}$ with respect to the operator $\mathscr{L}_{0}$ given by

$$
\mathscr{L}_{0} U_{2}=-\left(\mathscr{L}_{\mathrm{BS}(f(y))}-\mathscr{L}_{\mathrm{BS}(\bar{\sigma})}\right) U_{0} .
$$

Define a function $\phi$ by the solution of an ordinary differential equation given by

$$
\mathscr{L}_{0} \phi(y)=f(y)^{2}-\bar{\sigma}^{2} .
$$

Then we have

$$
\begin{aligned}
\mathscr{L}_{0} U_{2} & =-\left(\mathscr{L}_{\mathrm{BS}(f(y))}-\mathscr{L}_{\mathrm{BS}(\bar{\sigma})}\right) U_{0} \\
& =-\frac{1}{2}\left(f^{2}(y)-\bar{\sigma}^{2}\right) x^{2} \frac{\partial^{2} U_{0}}{\partial x^{2}} \\
& =-\frac{1}{2} \mathscr{L}_{0}^{-1}\left(f^{2}(y)-\bar{\sigma}^{2}\right) x^{2} \frac{\partial^{2} U_{0}}{\partial x^{2}} .
\end{aligned}
$$

Thus we obtain

$$
U_{2}(t, x, y, \tau)=-\frac{1}{2}(\phi(y)+k(t, x, \tau)) x^{2} \frac{\partial^{2} U_{0}}{\partial x^{2}}(t, x, \tau)
$$

for some $y$-independent function $k$.

On the other hand, applying the centering condition to the $\sqrt{\epsilon}$ term (31), we have a partial differential equation for $U_{1}$ given by

$$
\mathscr{L}_{\operatorname{Par}(\bar{\sigma})} U_{1}=-\mathscr{L}_{1} U_{2}
$$

Applying the operator $\mathscr{L}_{1}$ to (47), we obtain

$$
\begin{aligned}
\mathscr{L}_{\operatorname{Par}(\bar{\sigma})} U_{1}(l, x ; \tau)= & W_{2} x^{2} \frac{\partial^{2} U_{0}}{\partial x^{2}}(t, x, \tau) \\
& +W_{3} x^{3} \frac{\partial^{3} U_{0}}{\partial x^{3}}(t, x, \tau) .
\end{aligned}
$$

By adding the boundary condition (26), we obtain the desired result for $U_{2}$.

Using the similar argument as above, we have the result for $V_{1}$. 


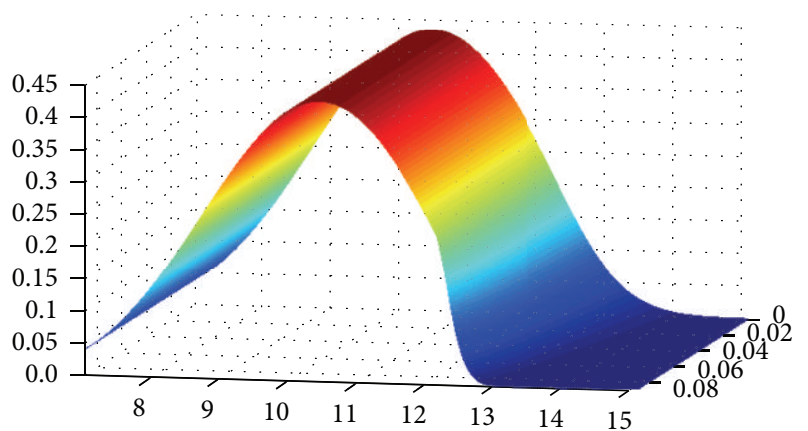

FIGURE 1: The leading order $P_{0}$ with respect to the price of underlying asset $X_{t}$ and the barrier time $\tau_{t}$.

\section{Computation}

This section shows the effect of the stochastic volatility on the Parisian option price through a numerical experiment.

In the previous section, we have derived the partial differential equations (35), (37), (40), and (43) for the leading order terms $U_{0}$ and $V_{0}$ and the correction terms $U_{1}$ and $V_{1}$, respectively. So, we have an approximation of the Parisian option price $P^{\epsilon}(12)$ in the form

$$
\begin{aligned}
P^{\epsilon}(t, x, y, \tau) & \approx P_{0}(t, x, \tau)+\sqrt{\epsilon} P_{1}(t, x, \tau) \\
& :=P_{0}(t, x, \tau)+\widetilde{P}_{1}(t, x, \tau),
\end{aligned}
$$

where the leading order term $P_{0}$ and the correction term $P_{1}$ are given by

$$
\begin{aligned}
& P_{0}(t, x, \tau)= \begin{cases}U_{0}(t, x, \tau), & \text { on } \mathscr{A}_{1}, \\
V_{0}(t, x), & \text { on } \mathscr{A}_{2},\end{cases} \\
& P_{1}(t, x, \tau)= \begin{cases}U_{1}(t, x, \tau), & \text { on } \mathscr{A}_{1}, \\
V_{1}(t, x), & \text { on } \mathscr{A}_{2},\end{cases}
\end{aligned}
$$

respectively.

We use the finite difference method (more concretely, the Crank-Nicolson scheme) to solve the partial differential equations. The parameters are set by $B=12, T=1, t=0$, $D=0.1, K=10, \bar{\sigma}=0.2, r=0.05, W_{2}=-0.00001$, $W_{3}=0.0041$, and $\epsilon=0.0001$.

Figure 1 shows the leading order term $P_{0}$ with respect to the price $X_{t}$ (the range of $X_{t}$ is from 6 to 15) and the barrier time $\tau_{t}$ (the range of $\tau_{t}$ is from 0 to $D=0.1$ ). As the feature of Up and Out type option, one can observe that the option price is almost zero when the price $X_{t}$ is bigger than the barrier $B$. Also the leading order term $P_{0}$ has the highest value near the strike price $K$, while the delta of $P_{0}$ is almost zero near the strike price $K$.

Figure 2 shows the correction term $\widetilde{P}_{1}$ with respect to the price $X_{t}$ (the range of $X_{t}$ is from 6 to 15) and the barrier time $\tau_{t}$ (the range of $\tau_{t}$ is from 0 to $D=0.1$ ). One can observe that the correction term $\widetilde{P}_{1}$ has a big fluctuation near the strike price $K$. The interesting feature is that the delta of $P_{1}$ is instantaneously very high near the strike price $K$. Clearly, it shows a different aspect of the stochastic volatility model in comparison with the Black-Scholes model.

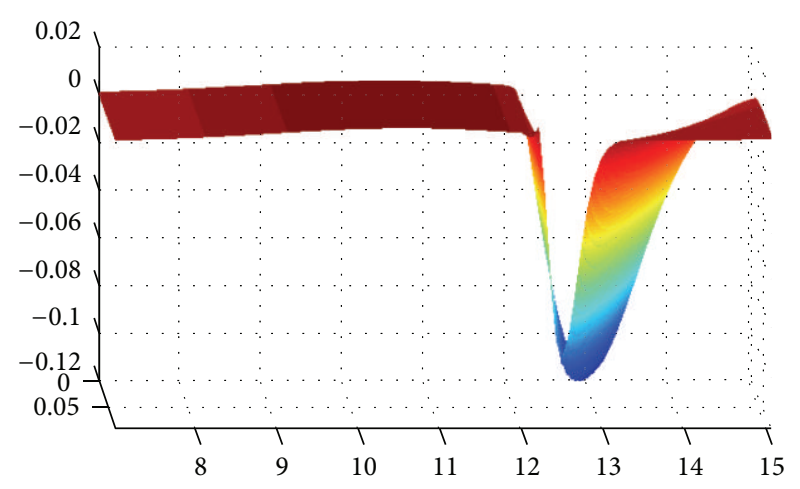

FIgURE 2: The correction term $\widetilde{P}_{1}$ with respect to the price of underlying asset $X_{t}$ and the barrier time $\tau_{t}$.

\section{Conclusion}

Based on the shortcomings of the Black-Scholes model, we choose a stochastic volatility model for the underlying asset price and study the pricing of a Parisian option. Under the fast mean-reverting stochastic volatility assumption, we approach the problem asymptotically and obtain equations for the approximated price and gain the price numerically by using the finite difference method. A remarkable feature is found as a correction effect of the stochastic volatility to the Black-Scholes model near the barrier. It is observed that the correction term is negative and has a hump shape around the barrier. So, the price of the Parisian option under the stochastic volatility model is underpriced compared to the price under the Black-Scholes model, particularly, near the barrier.

\section{Conflict of Interests}

The authors declare that there is no conflict of interests regarding the publication of this paper.

\section{References}

[1] F. Black and M. Scholes, "The pricing of options and corporate liabilities," Journal of Political Economy, vol. 81, pp. 637-654, 1973.

[2] J. Cox, "Notes on option pricing I: Constant elasticity of variance diffusions," Working Paper, Stanford University, 1975, (Reprinted in J. Portf. manage. vol. 22, pp. 15-17, 1996).

[3] S. L. Heston, "Closed-form solution for options with stochastic volatility with applications to bond and currency options," The Review of Financial Studies, vol. 6, no. 2, pp. 327-343, 1993.

[4] J.-P. Fouque, G. Papanicolaou, and R. Sircar, "Asymptotics of a two-scale stochastic volatility model," in Equations Aux Derivees Partielles et Applications, articles dedies a J.-L. Lions, pp. 517526, Gauthier-Villars, Paris, France, 1998.

[5] P. Carr, H. Geman, D. B. Madan, and M. Yor, "Stochastic volatility for Levy processes," Mathematical Finance, vol. 13, no. 3, pp. 345-382, 2003.

[6] R. J. Haber, P. J. Schonbucher, and P. Wilmott, "Pricing parisian options,” The Journal of Derivatives, vol. 34, pp. 71-19, 1999. 
[7] A. Marco and W. Lixin, "Pricing Parisian-style options with a lattice method," International Journal of Theoretical and applied Finance, vol. 2, no. 1, pp. 1-16, 1999.

[8] C. Labart and J. Lelong, "Pricing Parisian options using Laplace transforms," Bankers Markets and Investors, vol. 99, 24 pages, 2009.

[9] S.-P. Zhu and W.-T. Chen, "Pricing parisian and Parasian options analytically," Journal of Economic Dynamics \& Control, vol. 37, no. 4, pp. 875-896, 2013.

[10] L. B. Andersen and V. V. Piterbarg, "Moment explosions in stochastic volatility models," Finance and Stochastics, vol. 11, no. 1, pp. 29-50, 2007.

[11] J. P. Fouque, G. Papanicolaou, R. Sircar, and K. Solna, Multiscale Stochastic Volatility for Equity, Interest Rate and Credit Derivatives, Cambridge University Press, Cambridge, UK, 2011. 


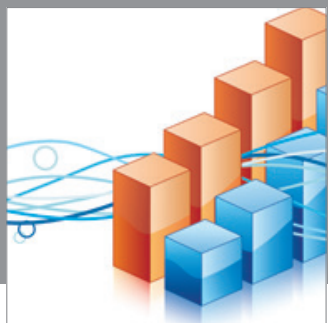

Advances in

Operations Research

mansans

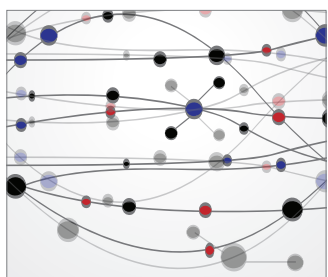

The Scientific World Journal
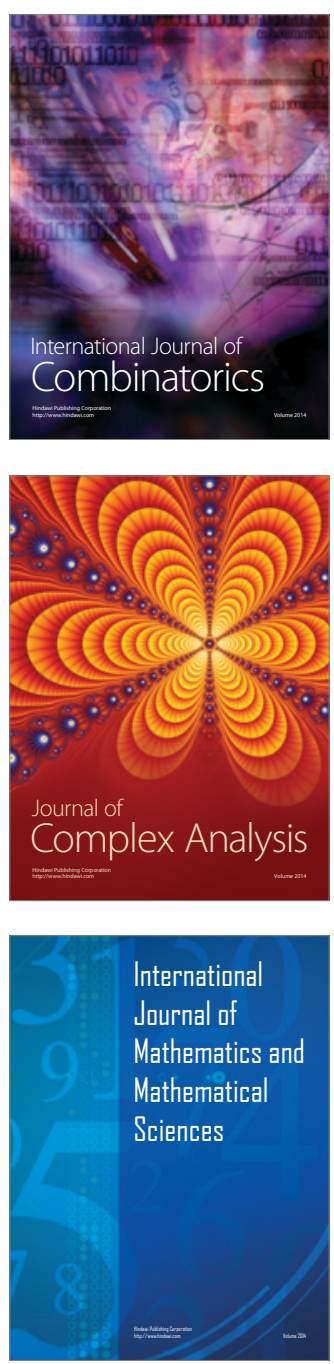
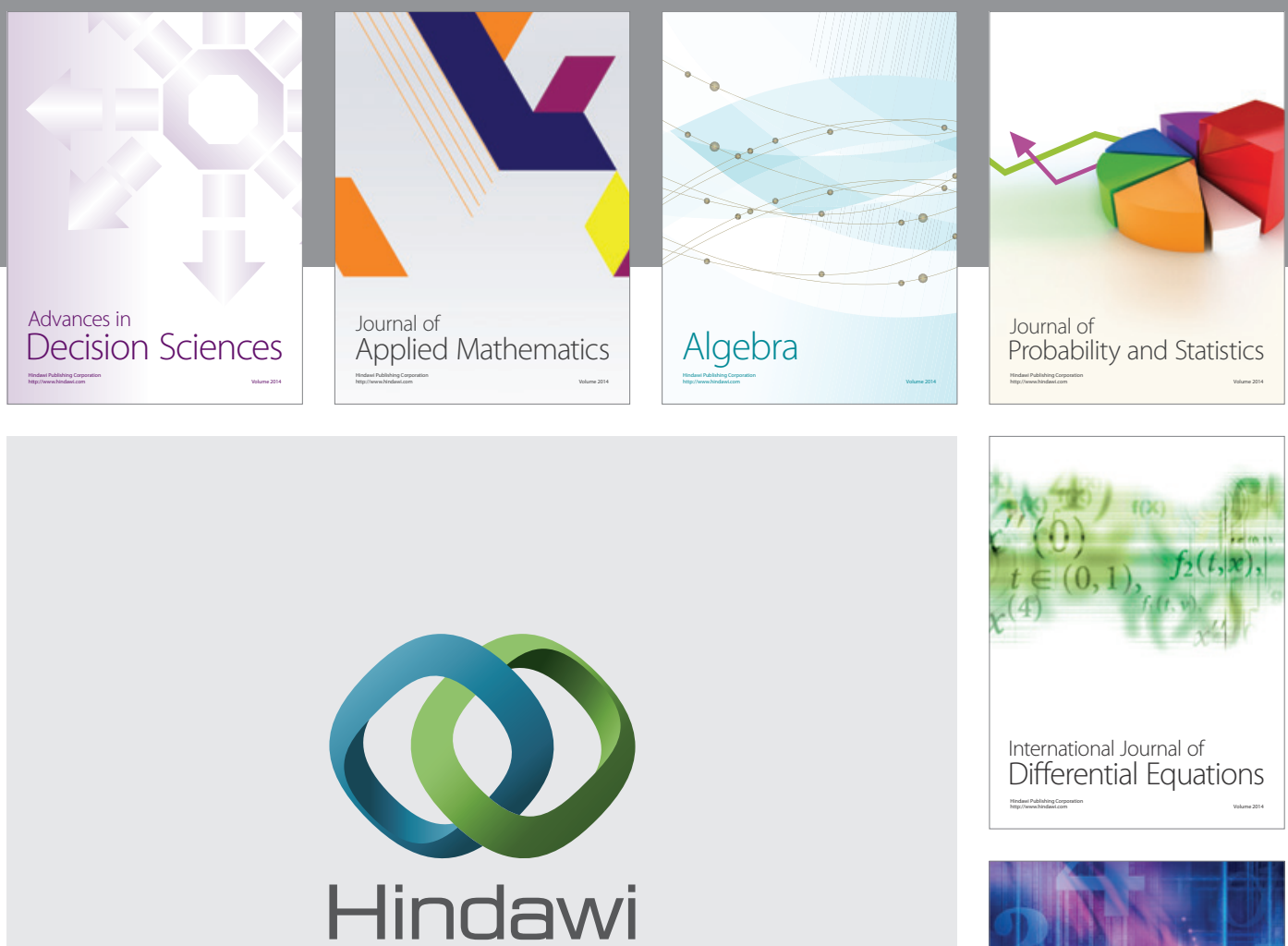

Submit your manuscripts at http://www.hindawi.com
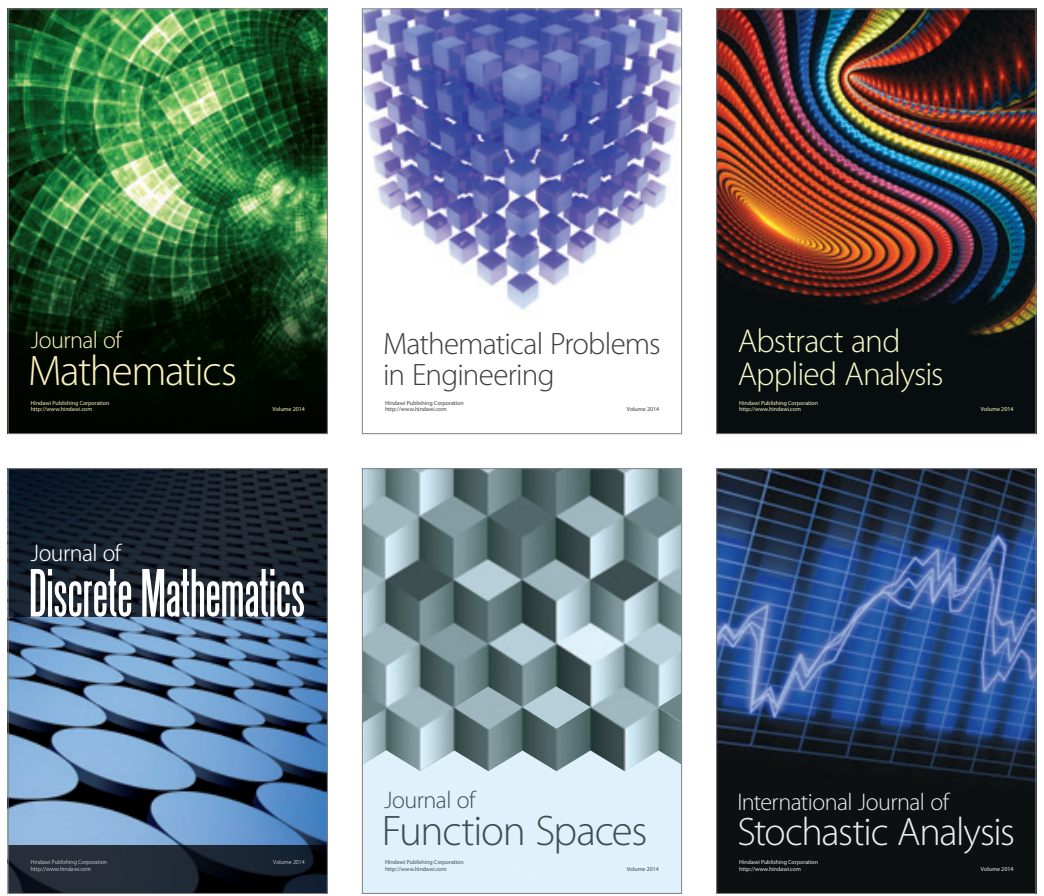

Journal of

Function Spaces

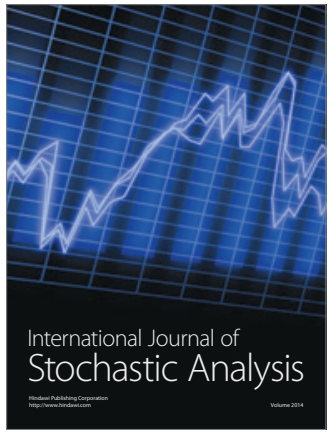

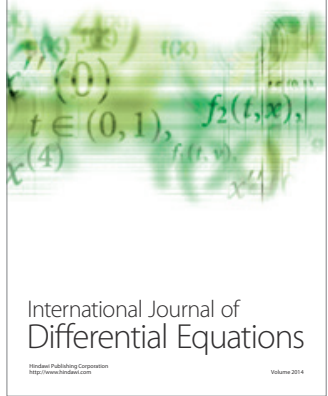
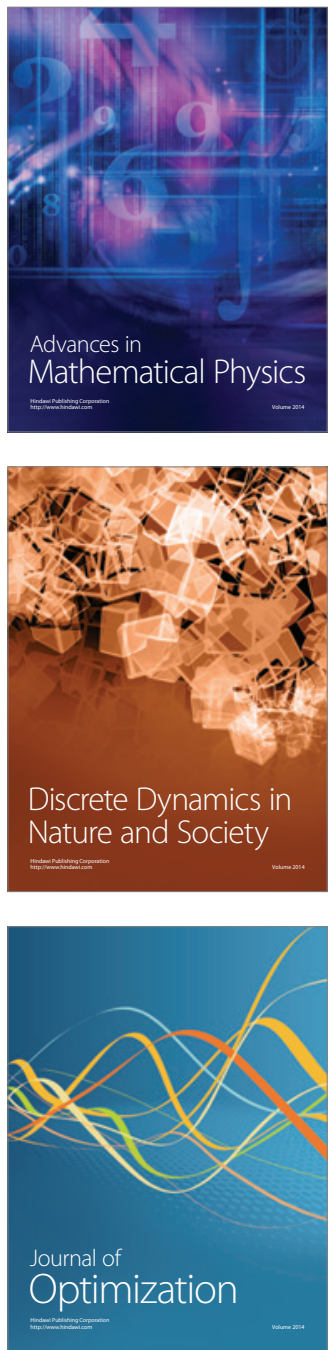